\title{
Evaluation of Ares-I Control System Robustness to Uncertain Aerodynamics and Flex Dynamics
}

\author{
Jiann-Woei Jang ${ }^{1}$, Chris Van Tassell ${ }^{2}$, Nazareth Bedrossian ${ }^{3}$ \\ The Charles Stark Draper Laboratory, Inc. \\ Charles Hall ${ }^{4}$ \\ NASA Marshall Space Flight Center \\ Pol Spanos ${ }^{5}$ \\ Department of Mechanical Engineering \& Material Science, Rice University
}

\begin{abstract}
This paper discusses the application of robust control theory to evaluate robustness of the Ares-I control systems. Three techniques for estimating upper and lower bounds of uncertain parameters which yield stable closedloop response are used here: (1) Monte Carlo analysis, (2) $\mu$ analysis, and (3) characteristic frequency response analysis. All three methods are used to evaluate stability envelopes of the Ares-I control systems with uncertain aerodynamics and flex dynamics. The results show that characteristic frequency response analysis is the most effective of these methods for assessing robustness.
\end{abstract}

\section{Nomenclature}

$\begin{array}{ll}\mu & =\text { structured singular value } \\ \mathrm{SSV} & =\text { structured singular value } \\ \mathrm{CLV} & =\text { crew launch vehicle } \\ \mathrm{CEV} & =\text { crew exploration vehicle } \\ \mathrm{LFT} & =\text { linear fractional transformation } \\ \boldsymbol{\Delta} & =\text { block diagonal perturbation matrix } \\ \boldsymbol{\Delta}_{\text {stab }} & =\text { maximum stabilizing perturbation matrix } \\ \beta & =\text { peak structured singular value } \\ k_{\mu} & =\text { structured singular value perturbation gain } \\ k_{G} & =\text { characteristic frequency response perturbation gain } \\ \lambda & =\text { system center scaling factor } \\ \gamma & =\text { structured singular value iteration step size } \\ \mathrm{MIMO} & =\text { multi-input, multi-output } \\ G(j \omega) & =\text { open loop transfer function } \\ \mathrm{OLTF} & =\text { open loop transfer function } \\ \mathrm{CLTF} & =\text { closed loop transfer function }\end{array}$

\footnotetext{
${ }^{1}$ Senior Member Technical Staff, Draper Laboratory, Houston, TX 77058; jjang@draper.com

2 Draper Fellow, Draper Laboratory, Draper Laboratory, Houston, TX 77058, cvantassell@draper.com

${ }^{3}$ Group Leader, Manned Space Systems; Draper Laboratory, Houston TX, 77058: naz@draper.com

${ }^{4}$ Senior Aerospace Engineer; NASA Marshall Space Flight Center, Huntsville, AL 35812:Charles.E.Hall@nasa.gov

${ }^{5}$ L. B. Ryon Professor of Mechanical and Civil Engineering, Rice University, TX 77005: spanos@rice.edu
} 


\section{Introduction}

The Ares-I autopilot is a complex, time-varying control system. Mass properties and aerodynamic forces vary considerably over the launch timeline. In addition, propellant slosh varies during upper stage flight as propellant is consumed. The autopilot's robustness in the presence of parameter uncertainty must be accurately evaluated to provide a precise stability envelope for the launch vehicle control systems. The stability envelope can be used as a yardstick for system engineers to determine if control system redesign is necessary for a given set of flight parameter variations such as aerodynamics and flex characteristics.

The most common method for assessing control systems' robustness is Monte Carlo analysis ${ }^{11}$, which is essentially a repeated guess and check screening process. Uncertainty in parameter values is modeled as a variation from nominal. Random samples of these parameters are taken to form unique system configurations or dispersions. The stability of each dispersion is determined through eigenanalysis, resulting in a map delineating stabilizing and destabilizing system configurations.

An alternative way to handle uncertainty in system parameters is by developing an uncertain system model using robust control theory ${ }^{2-9}$. Unlike the Monte Carlo approach, in which the system is re-formed for each set of parameter variations, the uncertain system model is formed only once. Wise considers several such techniques as applied to a simplified missile autopilot system in his paper on robustness tests ${ }^{9}$. In this paper, $\mu$ analysis and characteristic frequency response analysis are used to estimate the perturbations that the system can tolerate before becoming unstable. An iterative $\mu$ analysis algorithm is proposed for reducing conservatism when estimating bounds on uncertain parameters. Though certainly requiring fewer system formulations than Monte Carlo analysis, the algorithm's iterative nature makes it costly in terms of computation and time. This provides motivation to develop an even more efficient method of analysis-characteristic frequency response analysis ${ }^{6}$. The characteristic frequency response method is the multi-input, multi-output (MIMO) version of Bode analysis. This algorithm represents a major improvement over other MIMO analysis methods such as Monte Carlo or $\mu$ in that stability bounds can be determined using a single system formulation.

In this paper, all three methods are used to evaluate stability envelopes of the Ares-I control systems with uncertain aerodynamics and flex dynamics. The iterative $\mu$ analysis technique offers a systematic way to detect stability bounds. Unlike Monte Carlo, which randomly selects parameter values, $\mu$ analysis uses variable step sizes in an optimized directional search. Since $\mu$ requires that the system be evaluated at frequencies across the spectrum of interest, each iteration involves multiple system realizations. Depending on how quickly the $\mu$ algorithm converges, it may require more or less time than comparable Monte Carlo analysis. In the Ares-I case study, $\mu$ analysis takes longer than Monte Carlo. The characteristic frequency response algorithm is significantly faster than $\mu$ or Monte Carlo. This is because no iteration or random sampling is required. The basic algorithm is the fastest of those considered here and is excellent for obtaining a "first blush" estimate on stability bounds. In some systems, this estimate will reflect the actual bounds. However, in problematic systems that exhibit coupling between uncertain parameters, the modified algorithm can be used to accurately assess bounds. This carries the added benefit of differentiating between bounds on each parameter. This unique aspect of the 
characteristic frequency response algorithm makes it a valuable tool in system design and sets it apart from many other robustness tests.

\section{Monte Carlo Analysis}

The most common method for assessing robustness, Monte Carlo analysis is essentially a repeated guess and check analysis procedure. Uncertainty in parameter values is modeled as a variation from nominal. Random samples of these parameters are taken to form unique system configurations or dispersions. The stability of each dispersion is determined through eigenanalysis, resulting in a map delineating stabilizing and destabilizing system configurations.

The first step in performing Monte Carlo analysis is to identify the uncertain parameters to be studied. All other system parameters are held fixed while uncertain parameters are allowed to vary over specified ranges. Though any distribution may be used, uniform distribution is generally preferred because its lack of bias helps ensure adequate representation of the extreme values in the range. In this paper, all distributions are presumed to be uniform.

Each uncertain parameter forms an axis in the sample space. The boundaries of the sample space are defined by the range of variation for the respective parameter. Consider the spring mass damper system shown in Fig. 1a. Suppose there is some uncertainty in its stiffness and damping. These parameters form axes in the sample space. For demonstration purposes, stiffness and damping ranges are allowed to include negative values. The resulting sample space is depicted in Fig. 1b.

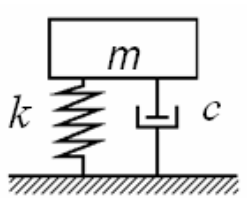

a)

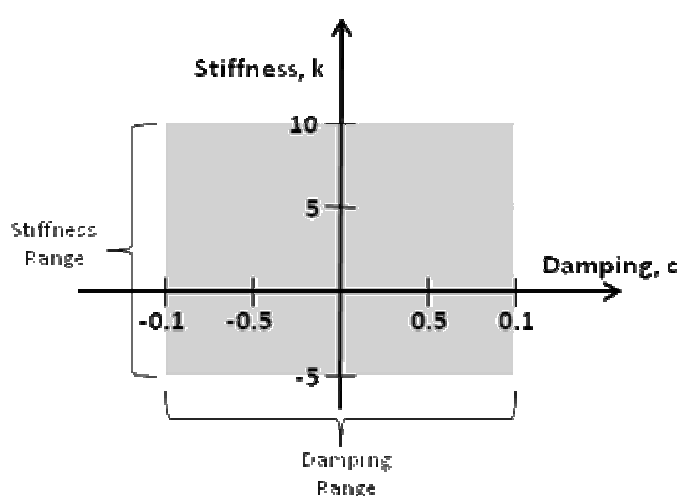

b)

Fig. 1 Spring Mass Damper (a) and Corresponding Sample Space (b)

As the number of uncertain parameters increases, the process is repeated to form a hyper cubic sample space. The uniform distribution of each parameter leads to an unbiased joint distribution in the sample space.

Next, parameter values are randomly selected from the sample space. A unique dispersion is formed for each sampling. Each dispersion represents a possible system configuration within the 
specified parameter ranges. The stability of these dispersions is then assessed through eigenanalysis. Finally, unstable dispersions are mapped into the sample space by plotting points representing their unique parameter combinations. The resulting scatter plot is a map of unstable regions (and conversely, stable regions) of the sample space. Fig. 2 shows the results of Monte Carlo analysis on the spring mass damper system from Fig. 1.

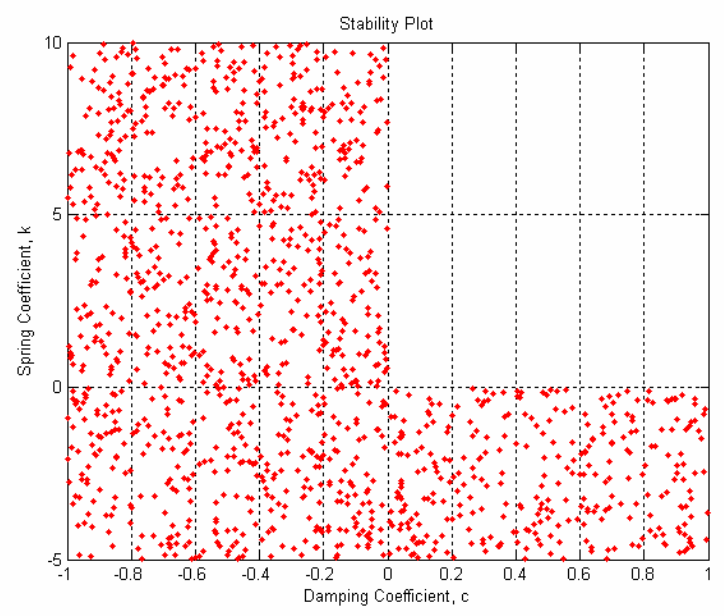

Fig. 2 Monte Carlo Scatter Plot

As expected, Fig. 2 shows that negative values of damping and stiffness cause instability.

\section{Uncertain System Model}

An alternative way to handle uncertainty in system parameters is by developing an uncertain system model. Unlike the Monte Carlo approach, in which the system is re-formed for each set of parameter variations, the uncertain system model is formed only once. Then, one of several methods is used to estimate the perturbations that the system can tolerate before becoming unstable. Two of these methods, $\mu$ analysis and characteristic frequency response, are outlined below.

The basis of the uncertain system model lies in representing uncertainty as a perturbation from nominal. Consider a generic uncertain parameter $a$ with nominal value $a_{0}$. Variations from nominal are represented by $\delta$ such that

$$
a=a_{0}+\delta
$$

where $\delta$ can assume positive or negative values. This formulation applies to both scalar and matrix parameters. Returning to the previously described spring mass damper system from Fig. 1a, uncertainty in $c$ and $k$ can be represented as perturbations from nominal. The resulting system is depicted in Fig. 3a. This uncertain system can also be represented in block diagram form as shown in Fig. 3b. 


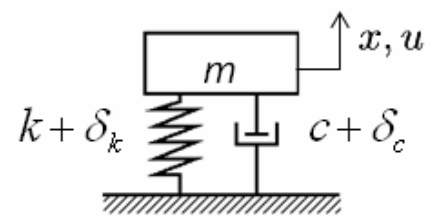

a)

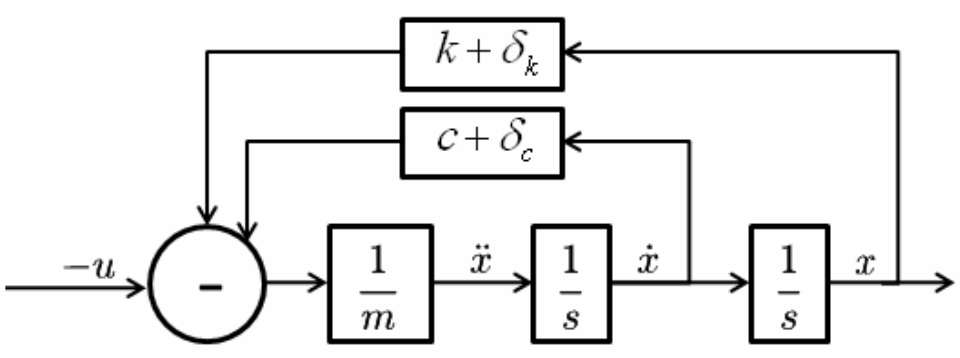

b)

Fig. 3 Uncertain Spring Mass Damper and Corresponding Block Diagram

The process of representing uncertain parameters as perturbations from nominal is helpful in visualizing sources of uncertainty in the system. However, its use is limited because perturbations are embedded in the system structure. In order to be more useful for robustness testing, perturbations (and hence uncertainty) must be separated from the rest of the system. One way to accomplish this is by replacing uncertain block elements with equivalent structures known as linear fractional transformations (LFTs) ${ }^{2-4}$.

Consider a generic uncertain block a representing Eq. (1) with input $u$ and output $y$. The resulting expression for $y$ is

$$
y=\left(a_{0}+\delta\right) u .
$$

The equivalent LFT representation of $\mathbf{a}$ is given by Fig. 4

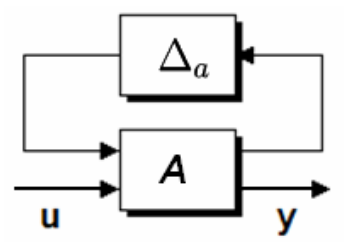

\section{Fig. 4 Linear Fractional Transformation (LFT) of a}

where $\mathbf{A}$ is a partitioned matrix and $\Delta_{a}$ is a matrix or scalar (depending on the structure of the uncertain parameter). A new expression is found for $y$ in terms of $\mathbf{A}$ and $\Delta_{a}$ as follows ${ }^{2-4}$

$$
y=\left(A_{22}+A_{21} \Delta_{a}\left(\mathrm{I}-A_{11} \Delta_{a}\right)^{-1} A_{12}\right) u,
$$

where $\Delta_{a}$ and the elements of $\mathbf{A}$ are selected such that Eq. (3) is equivalent to Eq. (2).

This process is repeated for every uncertain parameter and the resulting perturbation blocks are collected in the block diagonal structure $\boldsymbol{\Delta}$ such that $\boldsymbol{\Delta}=\operatorname{diag}\left(\Delta_{1}, \Delta_{2}, \ldots, \Delta_{\mathrm{n}}\right)$. This separates the perturbations from the rest of the system, M, leading to the familiar block diagonal uncertainty formulation shown in Fig. 5. 


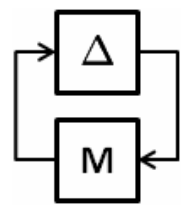

\section{Fig. 5 Block Diagonal Uncertain System Formulation}

The above process can be applied to systems of varying size and complexity. Though some effort is initially required to build the uncertain system model, once developed it has several applications, including $\mu$ analysis and characteristic frequency response techniques for assessing robustness. These techniques are described in the following sections.

\section{V. $\mu$ Analysis}

Having formulated the uncertain system model in such a way that uncertainty is isolated from the rest of the system, the next step is determining the range of allowable perturbations to maintain stability. Conversely, this process can be thought of as determining the smallest gain applied to $\Delta$ that drives the system unstable ${ }^{2}$. This is done using $\mu(M)$, the structured singular value (SSV) of M, which is defined as

$$
\frac{1}{\mu(M)}=\inf _{\Delta \in \underline{\Delta}}\{\bar{\sigma}(\Delta) \text { s.t.det }(I-\Delta M)=0\}
$$

where $\underline{\Delta}$ is the set of all possible $\Delta^{5}$. If no $\Delta$ satisfies $\operatorname{det}(I-\Delta M)=0$, then $\mu(M)=0$.

The first step in determining $\mu(M)$ is to evaluate $M(j \omega)$ at each frequency of interest in the spectrum over which the system operates. Next, $1 / \mu(M)$, which corresponds to the smallest $\Delta$ that destabilizes the system, is found at each frequency. Several popular algorithms, most employing an optimization scheme involving a search for suitable perturbations, exist for this purpose $^{3}$.

Next, $\mu(M)$ is plotted as a function of frequency and the peak value $\beta$ is determined. Using $\beta$, a test developed by Doyle ${ }^{3}$ and modified by Safonov ${ }^{6}$ shows that for any stabilizing $\Delta$,

$$
\bar{\sigma}(\Delta)<k_{\mu}
$$

where

$$
k_{\mu}=\frac{1}{\beta}
$$


This test can provide an estimate of the maximum allowable perturbation $\Delta_{\text {stab }}$ by applying the gain $k_{\mu}$ to the current perturbation $\Delta$ such that

$$
\Delta_{s t a b}=k_{\mu} \Delta
$$

Here, the form of the perturbation must be considered. Perturbations can take on any value, including complex values. In practice, many perturbations are strictly real. However, the SSV function may contain discontinuities when only real perturbations are considered ${ }^{7}$. This problem can be alleviated by considering complex perturbations. Though the SSV function obtained from complex perturbations is continuous, its results are generally conservative ${ }^{7}$.

In order to reduce conservatism, uncertain systems are "re-centered" by shifting the nominal values of uncertain parameters up or down. Consider a scaling factor $\lambda$ applied to the nominal values of uncertain parameters such that

$$
a=\lambda a_{0}+\delta
$$

Using this formulation, the system can be re-centered by simply increasing or decreasing $\lambda$. This re-centering can be visualized by plotting the system's center in the $s$-plane in terms of $\lambda$ such that $\lambda=1$ represents the original system, $\lambda>1$ shifts the system up, and $\lambda<1$ shifts the system down. Strictly real perturbations appear as lines on the real axis extending outward from system center. Complex perturbations are represented by circles around the system center, as shown in Fig. 6.

The first step in re-centering is to set $\lambda_{0}=1$ (corresponding to the original uncertain system) and perform $\mu$ analysis. The resulting $k_{\mu}$, which determines how much $\Delta$ can vary before driving the system unstable, defines a stable region around the system center as shown in Fig. 6a. Next, a new system center $\lambda_{1}$ is selected from within the stable region. If attempting to determine an upper bound, $\lambda_{1}$ is placed on the real axis near the right edge of the stable region, whereas the opposite is true when evaluating the lower bound. This process can be formalized as follows

$$
\begin{gathered}
\lambda_{k}=\left\{\begin{array}{cc}
1 & \text { for } k=0 \\
\prod_{i=1}^{k}\left(1+\gamma k_{\mu_{i}}\right) & \text { for } k=1,2, \ldots, n
\end{array}\right. \\
-1 \leq \gamma \leq 1
\end{gathered}
$$

where $\gamma$ is a step size that also determines the direction of re-centering (i.e. $\gamma>0$ for upper bound search and $\gamma<0$ for lower bound search). Then, the system is re-formed and reevaluated. A new $k_{\mu}$ is obtained and the process is repeated until $k_{\mu}$ becomes negligible (see Fig. 6b). 


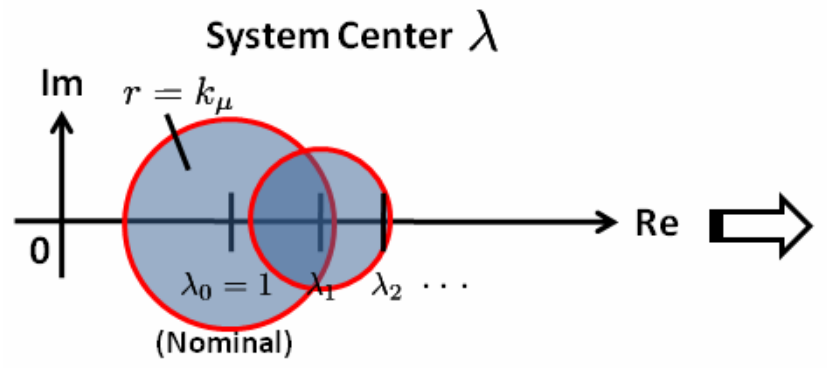

a)

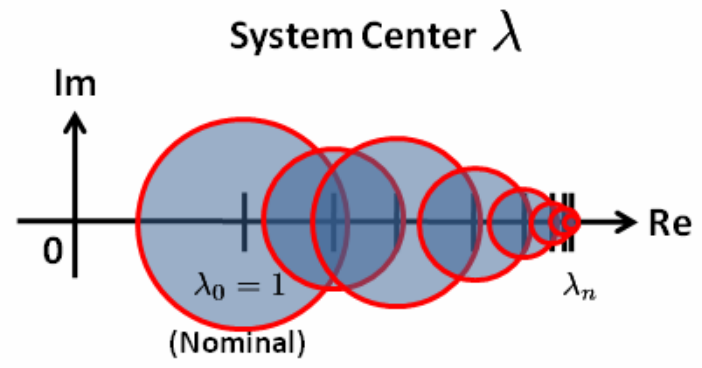

b)

Fig. 6 Re-centering of Uncertain System to Reduce Conservatism

The system center $\lambda_{n}$ at which $k_{\mu}$ is negligible defines the bound on uncertain parameters as follows

$$
\text { bound }=\lambda_{n}-1 \text {. }
$$

This $\mu$ analysis algorithm is very effective at reducing conservatism in estimates of bounds on uncertain parameters. However, it does not distinguish between parameters, i.e. it does not provide information about which parameter causes instability. Though certainly requiring fewer system formulations than Monte Carlo analysis, the algorithm's iterative nature makes it costly in terms of computation and time. This provides motivation to develop an even more efficient method of analysis - characteristic frequency response.

\section{Characteristic Frequency Response}

The characteristic frequency response method is the multi-input, multi-output (MIMO) version of Bode analysis. The basic algorithm represents a major improvement over other MIMO analysis methods such as Monte Carlo or $\mu$ in that stability bounds can be determined using a single system formulation. In addition, a modified characteristic frequency response algorithm, outlined in the next section, expands on the basic algorithm to address certain problematic systems and to identify bounds for each uncertain parameter individually.

\section{A. Basic Characteristic Frequency Response Algorithm}

Like Bode analysis, characteristic frequency response is performed on the open loop transfer function $G(j \omega)$. The closed loop uncertain system shown in Fig. 7a is re-cast in the familiar feedback form in Fig. 7b, with $G(j \omega)$ labeled accordingly. 


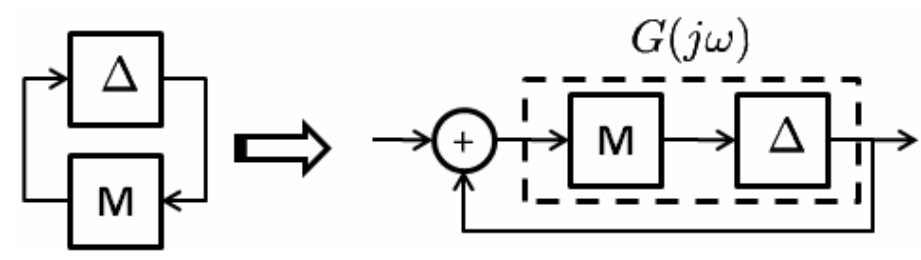

a)

b)

Fig. 7 Closed Loop Uncertain System in Original (a) and Feedback (b) Forms

The closed loop transfer function (CLTF) can also be written in terms of $G(j \omega)$ as follows

$$
C L T F=\frac{G(j \omega)}{1-G(j \omega)}
$$

Unlike Bode analysis, in which the overall system magnitude and phase are assessed at frequencies across the spectrum, characteristic frequency response calculates the magnitude and phase of each eigenvalue of the system at each frequency. The resulting eigenvalues are then ordered from most dominant to least dominant in magnitude. One way of simultaneously viewing both gain and phase of a particular response is in a Nyquist plot. This also provides the basis for stability analysis using the Nyquist criterion.

In its simplest form, the Nyquist criterion shows that Nyquist plots of the CLTF that encircle the origin indicate instability ${ }^{10}$. By considering the CLTF in terms of the open loop transfer function (OLTF), stability can be assessed using $G(j \omega)$. In the case of positive feedback, as shown in Eq. (11), $G(j \omega)$ encirclements of +1 are equivalent to CLTF encirclements of the origin. Similarly, $G(j \omega)$ encirclements of -1 are equivalent to CLTF encirclements of the origin in systems with negative feedback ${ }^{10}$. Referring to Fig. 7 and the description of the uncertain system model, positive feedback corresponds to positive perturbations while negative feedback represents negative perturbations.

The information in a Nyquist plot and the Nyquist criterion itself can be translated into appropriate forms for use in a Nichols plot. This is advantageous because Nichols plots are more intuitive than Nyquist plots and help spread the data out to form a clear picture of system behavior. The first step is to determine the gain and phase of the encirclement points. The point +1 occurs at unity magnitude and $0 \pm 360 k$ degrees of phase. Similarly, the point -1 occurs at unity magnitude and $180 \pm 360 k$ degrees of phase.

Next, consider a gain $k_{G}$ applied to $\Delta$. This represents a scaling factor on the perturbation. The original OLTF $G(j \omega)$ becomes $k_{G} G(j \omega)$ as shown in Fig. 8. 


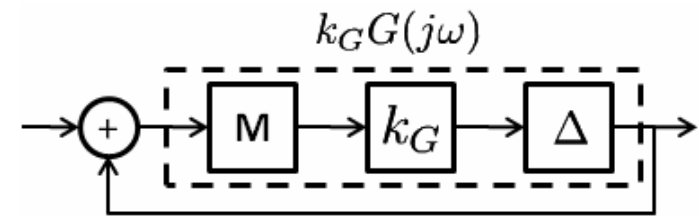

Fig. 8 Open Loop Transfer Function with Perturbation Gain $k_{G}$

Because the aforementioned encirclement points both have unity magnitude, the system must be stable if $k_{G}|G(j \omega)|<1$ at the phase corresponding to the encirclement points. In order to find the minimum destabilizing gain $k_{G, c r i t}$, this relationship is manipulated such that

$$
k_{G, c r i t}=\frac{1}{|G(j \omega)|} .
$$

Rather than plotting $|G(j \omega)|$ in the y-axis as in typical Nichols plots, $k_{G \text {,crit }}$ is plotted. Phase makes up the $\mathrm{x}$-axis as usual.

The points of interest are those where $k_{G, c r i t}$ crosses 0 or $180( \pm 360 k)$ degrees of phase. Hence forth, these phase crossings will be referred to as upper bound crossings and lower bound crossings, respectively. The value of $k_{G, \text { crit }}$ at an upper bound crossing indicates how much positive gain can be applied to the specified perturbation $\Delta$ before the system goes unstable. Conversely, $k_{G, \text { crit }}$ at a lower bound crossing is the amount of negative gain that drives the system unstable. When the plot intersects more than one upper or lower bound crossing, the minimum values (smallest absolute values) are used.

Using the previous example of a spring mass damper with uncertainty in stiffness and damping, eigenvalues of the open loop uncertain system are evaluated. In this case, there is only one significant response. Next, $k_{G, c r i t}$ is plotted vs. phase as shown in Fig. 9. 


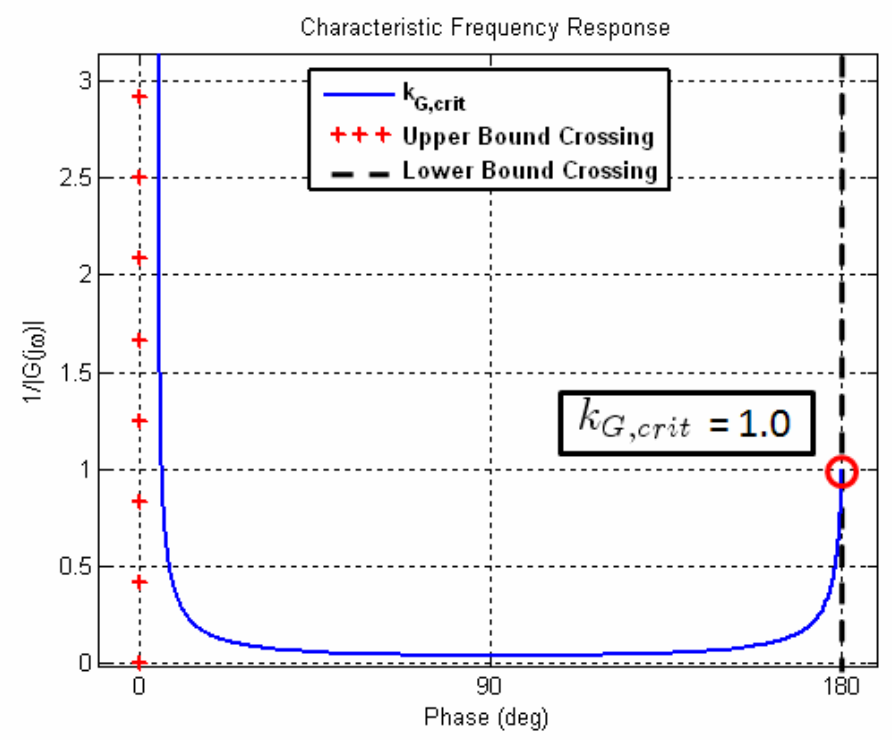

Fig. 9 Characteristic Frequency Response of Uncertain Spring Mass Damper

There is a lower bound crossing with $k_{G, c r i t}=1.0$ and no upper bound crossing. Hence, the system is stable for stiffness and damping variations between $-100 \%$ and $+\infty$. This matches the results of the Monte Carlo analysis and the intuitive result that the system remains stable as long as stiffness and damping are positive.

\section{B. Modified Characteristic Frequency Response Algorithm}

Though the basic characteristic frequency response algorithm is effective at determining upper and lower bounds on uncertain parameters, it does not distinguish between parameters. In other words, the bounds derived from the basic algorithm correspond to the minimum possible variation in any parameter that causes instability. Since $k_{G}$ is applied to all perturbations equally, the basic algorithm effectively performs a linear search in the parameter space with all parameters increasing or decreasing together. This is shown in Fig. 10, which depicts the effective search pattern corresponding to Fig. 9.

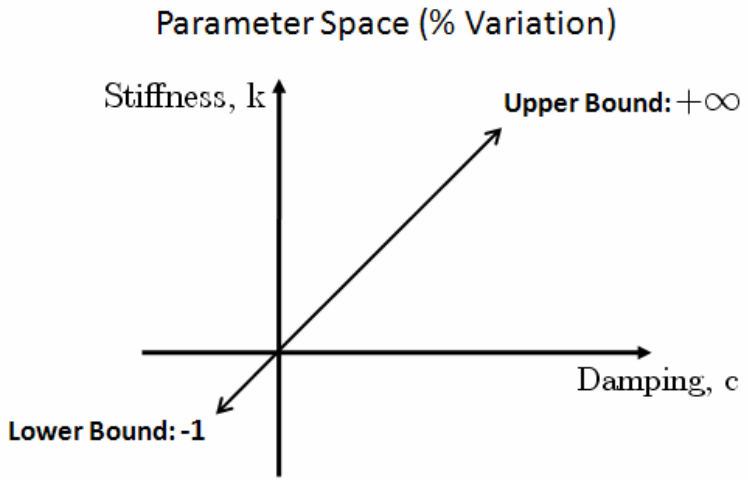

Fig. 10 Effective Search Pattern of Basic Algorithm 
The basic algorithm forms the first step of the modified algorithm. Having established upper and lower bounds for the entire system, the next step is to find specific bounds for each parameter. This is accomplished by selecting a bound and setting all but one of the uncertain parameters to that value. The result is a new system with a single uncertain parameter. The basic algorithm is used to evaluate this system, resulting in bounds specific to the uncertain parameter being examined. This process is repeated for all uncertain parameters and at both the upper and lower bounds.

\section{Case Study}

In order to assess the performance of the analysis methods outlined in the previous section, a case study is conducted using the Ares-I crew launch vehicle. Ares-I is a 2-stage rocket designed to carry the Orion crew exploration vehicle (CEV) to low-Earth orbit. Throughout its launch timeline, Ares-I experiences changes in both mass properties and environment. This case study employs the aforementioned analysis techniques to determine Ares-I's robustness to these changes.

In this case study, vehicle pitch is studied. Because Ares-I is nearly symmetric about its longitudinal axis, the results obtained from the pitch axis are equally applicable to yaw. Launch vehicle dynamics, including propellant slosh and structural bending, are described by Frosch and Vallely ${ }^{11}$. A PID controller is used as a stabilizing autopilot system. The resulting nominal system is cast in state space form such that

$$
\begin{aligned}
& \dot{x}=A x+B u \\
& y=C x+D u
\end{aligned}
$$

Next, uncertain system parameters are selected for study. Of the many parameters with some uncertainty, aerodynamic forces, $C_{z \alpha}$ and vehicle bending mode frequencies, $\omega_{b i}$ are among the most sensitive to perturbations. For modeling purposes, perturbations in $\omega_{b i}{ }^{2}$ are considered. Perturbations in these parameters are considered in the analysis that follows.

\section{A. Monte Carlo Analysis}

Monte Carlo analysis is performed using the nominal system model with many sampled parameter values from the sample space. In this case, the axes of the sample space are $C_{z \alpha}$ and

$\omega_{b i}{ }^{2}$, which range from nominal value $-80 \%$ to $+80 \%$ and $-125 \%$ to $+200 \%$, respectively. The stability scatter plot, shown in Fig. 11, is formed by analyzing 10,000 dispersions. The resulting stability bounds are $-100 \%$ to $+170 \%$ in $C_{z \alpha}$ and $-68 \%$ in $\omega_{b i}{ }^{2}$ (no upper bound exists). 


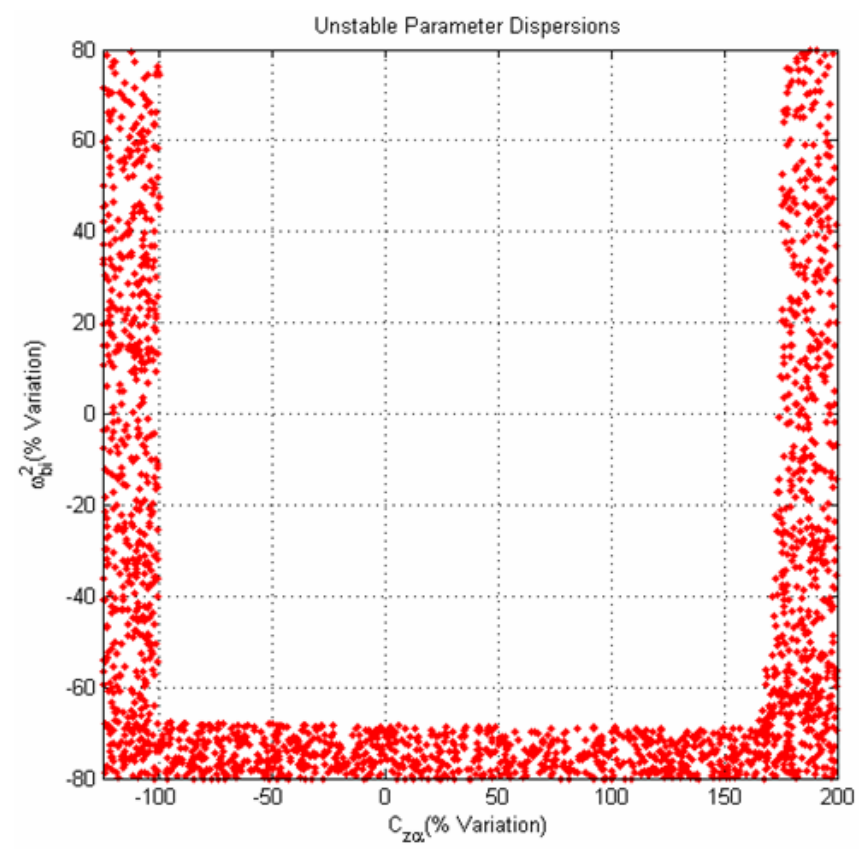

Fig. 11 Monte Carlo Scatter Plot for Uncertainty in Aerodynamics and Bending

\section{Frequencies}

\section{B. $\mu$ Analysis}

The uncertain system model, which separates perturbations in $C_{z \alpha}$ and $\omega_{b i}{ }^{2}$ from the rest of the system, is used when performing $\mu$ analysis. Using complex perturbations, the system center is initially scaled by $\lambda=1$ and $k_{\mu}$ is obtained through $\mu$ analysis. Then, $\lambda$ is decreased and the process is repeated until $k_{\mu}<0.01$. This occurs at $\lambda_{n}=0.33$, corresponding to a lower bound of $-67 \%$. The procedure is repeated, this time increasing $\lambda$ at each iteration to arrive at $\lambda_{n}=1.77$. The upper bound is thus $+177 \%$.

Comparison with the results obtained from Monte Carlo analysis reveals a problematic feature of the system. Unlike the spring mass damper used in previous examples, Ares-I exhibits crosscoupling between uncertain parameters. Careful examination of Fig. 11 shows that as $\omega_{b i}^{2}$ increases, the upper bound on $C_{z \alpha}$ increases. The effective search pattern for $\mu$ analysis is the same as for the basic characteristic frequency response algorithm, so both uncertain parameters increase and decrease together. The result is that the upper bound estimate produced by $\mu$ analysis is overly optimistic.

\section{Characteristic Frequency Response}

As in $\mu$ analysis, characteristic frequency response techniques are applied to the uncertain system model. Magnitude and phase of the eigenvalues of the uncertain system are evaluated, then ordered by magnitude. Next, $k_{G \text {,crit }}$ is plotted against phase for each of the dominant 
eigenvalues (in this case, there are 2). Upper and lower bounds are determined by taking the minimum value of $k_{G, c r i t}$ at upper bound crossings and lower bound crossings, as shown in Fig. 12. This analysis results in upper and lower bound estimates of $+177 \%$ and $-68 \%$, respectively.

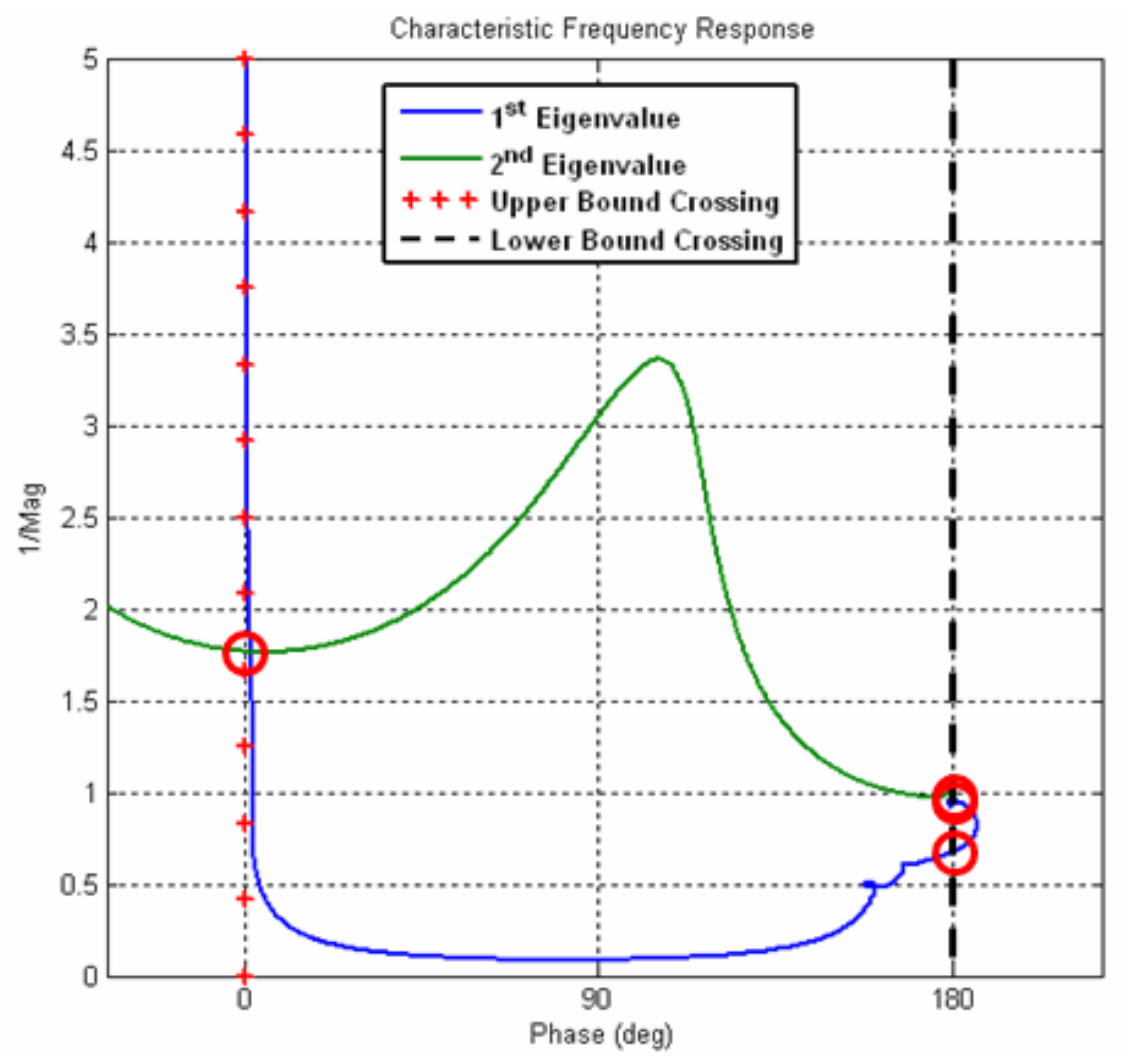

Fig. 12 Characteristic Frequency Response of Uncertain Ares-I Model

As with the upper bound obtained by $\mu$ analysis, the upper bound predicted by the basic characteristic frequency response algorithm is overly optimistic. Again, this is due to the algorithm's effective search pattern, which examines only the upper right and lower left corners of the parameter space. In order to explore all 4 corners of the parameter space, the modified algorithm is employed. This results in separate bounds of $-100 \%$ to $+171 \%$ in $C_{z \alpha}$ and $-68 \%$ in $\omega_{b i}^{2}$.

\section{Performance Summary}

Though the ultimate objective of all of the algorithms presented here is to identify bounds on stability, speed and computational demand are also useful in assessing the relative strengths and weaknesses of each algorithm. Here, computational demand is evaluated in terms of number of system formulations required. Table 1 summarizes the results of each algorithm's analysis of Ares-I using a Windows Vista PC running dual core $1.86 \mathrm{GHz}$ processors. 


\begin{tabular}{|c|c|c|c|c|}
\hline Method & $\begin{array}{c}\text { Number of } \\
\text { Systems Formed }\end{array}$ & $\begin{array}{c}\text { Time } \\
\text { (sec) }\end{array}$ & $\begin{array}{c}\text { Upper } \\
\text { Bound }\end{array}$ & $\begin{array}{c}\text { Lower } \\
\text { Bound }\end{array}$ \\
\hline Monte Carlo & 10,000 & 265 & $+170 \%$ & $-68 \%$ \\
\hline$\mu$ Analysis & 57 & 1,163 & $+177 \%$ & $-67 \%$ \\
\hline $\begin{array}{c}\text { Basic Char. } \\
\text { Freq. Response }\end{array}$ & 1 & 6 & $+177 \%$ & $-68 \%$ \\
\hline $\begin{array}{c}\text { Modified Char. } \\
\text { Freq. Response }\end{array}$ & 5 & 30 & $+171 \%$ & $-68 \%$ \\
\hline
\end{tabular}

Table 1 Summary of Analysis Results

The basic characteristic frequency response algorithm is both the fastest at 6 seconds and the least computationally demanding, requiring a single system formulation. However, like $\mu$ analysis, it overestimates the upper bound by $7 \%$. The modified characteristic frequency response algorithm requires more time and system formulations than the basic algorithm, but generates a more accurate upper bound.

\section{Conclusion}

In this paper, the characteristic frequency response algorithm and an iterative version of $\mu$ analysis were described and tested. Their performance was assessed with Monte Carlo analysis as a benchmark test. The iterative $\mu$ analysis technique offers a systematic way to detect stability bounds. Unlike Monte Carlo, which randomly selects parameter values, $\mu$ analysis uses variable step sizes in an optimized directional search. Since $\mu$ requires that the system be evaluated at frequencies across the spectrum of interest, each iteration involves multiple system realizations. Depending on how quickly the $\mu$ algorithm converges, it may require more or less time than comparable Monte Carlo analysis. In the above Ares-I case study, $\mu$ analysis takes longer than Monte Carlo.

The characteristic frequency response algorithm is significantly faster than $\mu$ or Monte Carlo. This is because no iteration or random sampling is required. The basic algorithm is the fastest of those considered here and is excellent for obtaining a "first blush" estimate on stability bounds. In some systems, this estimate will reflect the actual bounds. However, in problematic systems that exhibit coupling between uncertain parameters, the modified algorithm can be used to accurately assess bounds. This carries the added benefit of differentiating between bounds on each parameter. This unique aspect of the characteristic frequency response algorithm makes it a valuable tool in system design and sets it apart from many other robustness tests.

\section{Acknowledgments}

Acknowledgments go here. 


\section{References}

${ }^{1}$ Sobol', I.M., A Primer for the Monte Carlo Method, CRC Press, Boca Raton, 1994.

${ }^{2}$ Maciejowski, J. M., Multivariable Feedback Design. Addison-Wesley Publishing Company, Inc., New York, 1989.

${ }^{3}$ Balas, Gary, et al. Mu-Analysis and Synthesis Toolbox. The Math Works, Inc., Natick, 1998, Chap. 4.

${ }^{4}$ Zhou, K., Doyle, J.C., and Glover, K., Robust and Optimal Control, Prentice-Hall, Upper Saddle River, 1996

${ }^{5}$ Dailey, R. Lane. "Lecture Notes for the Workshop on H-Infinity and Mu Methods for Robust Control." 1991 IEEE Conference on Decision and Control. Institute of Electrical and Electronics Engineers, Inc., Brighton, 1991, pp. 45-81.

${ }^{6}$ Chiang, Richard and Michael Safonov. Matlab Robust Control Toolbox. The Math Works, Inc., Natick, 1997, Chap. 1.

${ }^{7}$ Packard, A and P. Pandey. "Continuity Properties of the Real/Complex Structured Singular Value." IEEE Transactions on Automatic Control Vol. 38, No. 3, 1993, pp. 415-428.

${ }^{8}$ Postma, B., Jang, J., Bedrossian, N., Spanos, P., "Robust Constrained Optimization Approach for International Space Station Centrifuge Rotor Auto-Balancing Controller," AIAA GN\&C Conference, August, 2005.

${ }^{9}$ Wise, Kevin A. "Comparison of Six Robustness Tests Evaluating Missile Autopilot Robustness to Uncertain Aerodynamics." Journal of Guidance, Control, and Dynamics Vol. 15, No. 4, 1992, pp. 861-870.

${ }^{10}$ Tilbury, Dawn and Luis J Oms. Frequency Response Tutorial, Nyquist. 27 August 1996. 3 January $2008<\mathrm{http}: /$ www.engin.umich.edu/group/ctm/freq/nyq.html $>$.

${ }^{11}$ Frosch, James and Donald Vallely. "Saturn AS-501/ S-IC Flight Control System Design." Journal of Spacecraft Vol. 4, No. 8, 1967, pp. 1003-1009. 